\title{
Study on Multi-Objective Optimization Design and Passive Control of Wind Turbine Airfoil
}

\section{Yong Peng ${ }^{1}$, Jun Wang ${ }^{1,2}$, Wei Wang ${ }^{2}$, Guoqing Yin ${ }^{1}$}

${ }^{1}$ China-EU Institute for Clean and Renewable Energy, Huazhong University of Science and Technology, Wuhan, 430074, 5 China

${ }^{2}$ School of Energy and Power Engineering, Huazhong University of Science and Technology, Wuhan, 430074, China

Correspondence: Jun Wang (wanghhust@163.com)

\begin{abstract}
In this paper, the class-shape function transform (CST) parametric method is used to parameterize the airfoil configuration, and a new airfoil is randomly generated within a limited range. The 2D Reynolds-Averaged Navier-Stokes (RANS) computational fluid dynamics (CFD) solver is used to compute the quantities such as lift-to-drag ratio. The multiobjective genetic algorithm performs multi-objective optimization design on the airfoil plane shape to achieve high lift-todrag ratio with low drag in operating ranges of angle of attack, and finally obtains the Pareto optimal solution set. The mixed function of index method is used to increase the thickness of the trailing edge of the airfoil. From the multi-objective solutions and blunt trailing edge solutions which represent the best trade-offs between the design objectives, one can select a

15 set of airfoil shapes with a low relative drag force and with improved aerodynamic performance. Taking a typical airfoil NACA4418 as an example. The results show that the optimized airfoil has a better pressure distribution than the original airfoil, effectively increasing the lift coefficient and reducing the drag coefficient. After thickening the trailing edge of the optimized airfoil, the results show that the lift coefficient is improved at all angles of attack and the stall is delayed. And the blunt trailing edge airfoil has better lift-to-drag characteristics than the original airfoil and the optimized airfoil.
\end{abstract}

20 Keywords. wind turbine, airfoil shape, aerodynamic performance, optimization design, blunt trailing edge 


\section{Introduction}

The aerodynamic performance of the airfoil directly affects the power generation capacity of the wind turbine. At present, the airfoil optimization design method can be roughly divided into an inverse design method and an optimized design method (Ma et al., 2010). The inverse design method uses a mathematical method to inversely derive an airfoil that satisfies

5 the target pressure distribution by using a velocity or pressure distribution corresponding to the target airfoil through geometric and flow control equations. The optimization design method is a global search optimization method. It directly uses the airfoil aerodynamic parameters such as the lift-to-drag ratio as the objective function, and through the correction of the airfoil geometry and the numerical optimization program to search the extremum of the objective function to complete the optimization design (Yan, 1999). Based on Eppler theory and inverse design method, Tangler and Somers designed 35

10 NREL-S airfoils which has good lift-to-drag ratio characteristics for various working conditions (Tangler and Somers, 1995). Based on CFD technology and numerical optimization method, the Ris $\varphi$ National Laboratory of Denmark has designed a series of wind turbine airfoils suitable for different operating conditions and different control modes with the maximum liftto-drag ratio as the optimization target (Fuglsang and Bak, 2004). James Ferguson (Ferguson, 1964), Hicks Henne (Hicks and Henne, 1978), Sobieczky (Sobieczky, 1999) and Kulfan (Kulfan and Bussoletti, 2006) have proposed different airfoil

15 shape representation methods. These parameterization methods combined with optimization methods such as Genetic Algorithm, Simulate Anneal Arithmetic and Complex Method greatly promote the efficiency of airfoil optimization.

In recent years, with the increasing capacity of wind turbines, and in view of the dual considerations of performance and structure, most of the blade airfoil types are blunt tailing edge airfoil (van Rooij and Timmer, 2003). Compared with the same maximum thickness of the trailing edge airfoil, the blunt trailing edge airfoil has a larger cross-sectional area and

20 higher bending and torsion resistance, which has a higher maximum lift coefficient, a larger stall angle of attack and a lower surface roughness sensitivity in aerodynamic performance (Jackson et al., 2005). The blunt tailing edge airfoil thickening methods mainly include direct truncation method (Law and Gregorek, 1987), symmetric thickening method (Standish and van Dam, 2003), asymmetric thickening method (Zhang, 2009) and airfoil rigid rotation method (Deng et al., 2011).

This paper combines airfoil optimization with passive control technology. Taking the NACA4418 airfoil as the original

25 airfoil, the multi-objective genetic algorithm is coupled with the class shape transformation (CST) method to optimize the wind turbine airfoil. And then the Mixed function of index method is used to asymmetrically thicken the optimized airfoil trailing edge to maximize the aerodynamic performance of the airfoil.

\section{Optimization Design Method}

Figure 1 is the flow chart of the optimization design method of this paper. In the first step, the parameterized airfoil is

30 generated by the CST method, and the grid division and numerical calculation are performed by calling the ICEM and FLUENT software through the scripts. In the second step, the genetic algorithm obtains the aerodynamic performance data and evaluates the fitness. 


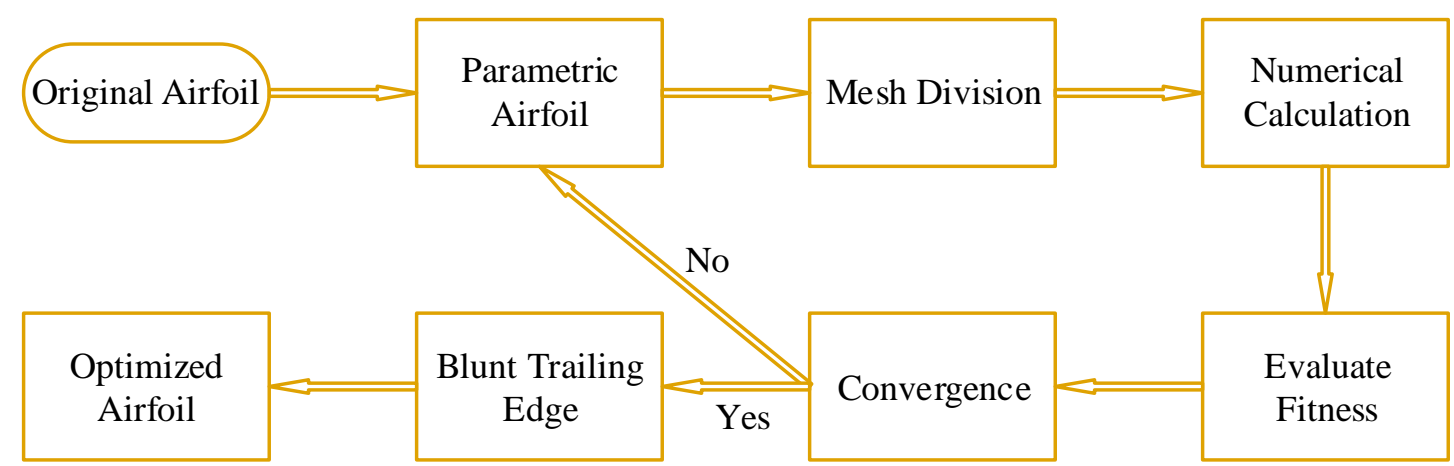

Figure 1: The flow chart of the optimization design method.

When the fitness function converges, an optimization result is obtained. Conversely, a new parametric airfoil is regenerated and the calculation is repeated until a series of pareto optimal solution sets are obtained. In the third step, the airfoil with good performance is used to passivate the trailing edge to further improve the aerodynamic performance of the airfoil.

\subsection{CST Parameterization}

Class Shape Transformation (CST) method has been proposed by Professor Kulfan (Kulfan and Bussoletti, 2006). This method was implemented in a tool that combined all aspects of the aerodynamic design process: parameterization, aerodynamic analysis, and optimization. The two-dimensional geometry by the product of the class function $\mathrm{C}(\mathrm{x})$, representing a specific class of shapes, and a shape function $\mathrm{S}(\mathrm{x})$, which defined the deviation from the class function, plus a term that characterizes the trailing edge thickness:

$z(x)=C(x) \cdot S(x)+(x) \cdot \Delta z_{t e}$,

The class function is given in generic form by:

$15 C(x) \equiv(x)^{N_{1}}(1-x)^{N_{2}}$ for $0 \leq x \leq 1$,

The exponents $\mathrm{N} 1$ and $\mathrm{N} 2$ range from 0 to 1 define the type of geometry to be represented. For an airfoil with a round leading edge and sharp trailing edge, the $\mathrm{N} 1$ and $\mathrm{N} 2$ are equal to 0.5 and 1.0, separately. By using Bernstein polynomial, the shape function can be used to generate arbitrary airfoil shape (Farouki, 2000). The definition of the shape function is:

$\left\{\begin{array}{l}S(x)=\sum_{i=0}^{n}\left[b_{i} \cdot K_{i, n} \cdot(x)^{i} \cdot(1-x)^{n-i}\right] \\ K_{i, n}=\frac{n !}{i !(n-i) !}\end{array}\right.$,

20 The leading edge radius of the airfoil, the boat-tail angle and the ordinate of the trailing edge point have the following relationship at the extremes of the interval $[0,1]$. 
$\left\{\begin{array}{l}S(0)=\sqrt{\frac{2 R_{l e}}{c}} \\ S(1)=\tan \beta+\frac{\Delta z_{t e}}{c}\end{array}\right.$,

where $b_{i}(i=0,1 \cdots)$ is the weight factors, represent the design parameters. The value of $b_{i}$ can be obtained as follows:

$\underbrace{\left[\begin{array}{cccc}S_{0}\left(x_{0}\right) & S_{1}\left(x_{0}\right) & \cdots & S_{n}\left(x_{0}\right) \\ S_{0}\left(x_{1}\right) & S_{1}\left(x_{1}\right) & \cdots & S_{n}\left(x_{1}\right) \\ \vdots & \vdots & \ddots & \vdots \\ S_{0}\left(x_{n}\right) & S_{1}\left(x_{n}\right) & \cdots & S_{n}\left(x_{n}\right)\end{array}\right]}_{M} \cdot\left[\begin{array}{c}b_{0} \\ b_{1} \\ \vdots \\ b_{n}\end{array}\right]=\underbrace{\left[\begin{array}{c}y\left(x_{0}\right) \\ y\left(x_{1}\right) \\ \vdots \\ y\left(x_{n}\right)\end{array}\right]}_{Y}$,

with $S_{i}(x)=C\left(x_{i}\right) \cdot K_{i, n} \cdot(x)^{i}(1-x)^{n-i}, x_{i} \in(0,1), i=0,1 \cdots$

5 Knowing the geometric shape of the control point $\left(x_{i}, \mathrm{y}\left(x_{i}\right)\right)$, the uniqueness of the CST parameterization method can be verified according to equation (5). In addition, the small fluctuations of the parameterized matrix $\mathrm{M}$ and the column vector $\mathrm{Y}$ cause a large change in the weight factor, and vice versa.

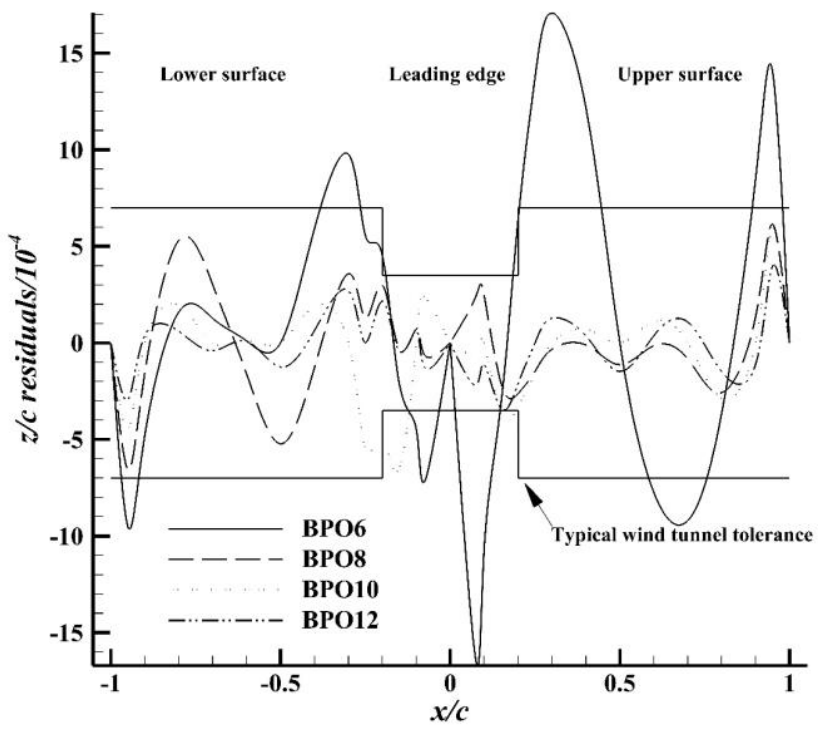

Figure 2: Residuals comparison of different Bernstein polynomial orders (BPO).

In order to verify the accuracy of the parameterization method, Figure 2 shows the residual comparison of different Bernstein polynomial orders (BPO). It can be seen that BPO8, BPO10 and BPO12 have higher accuracy, however the higher polynomial order will occupy more computation resources. Therefore, BPO8 is chosen to fit the original airfoil. Figure 3 shows a comparison of the BPO8 parametric airfoil with the initial airfoil. It can be seen that the two airfoils are substantially coincident. 


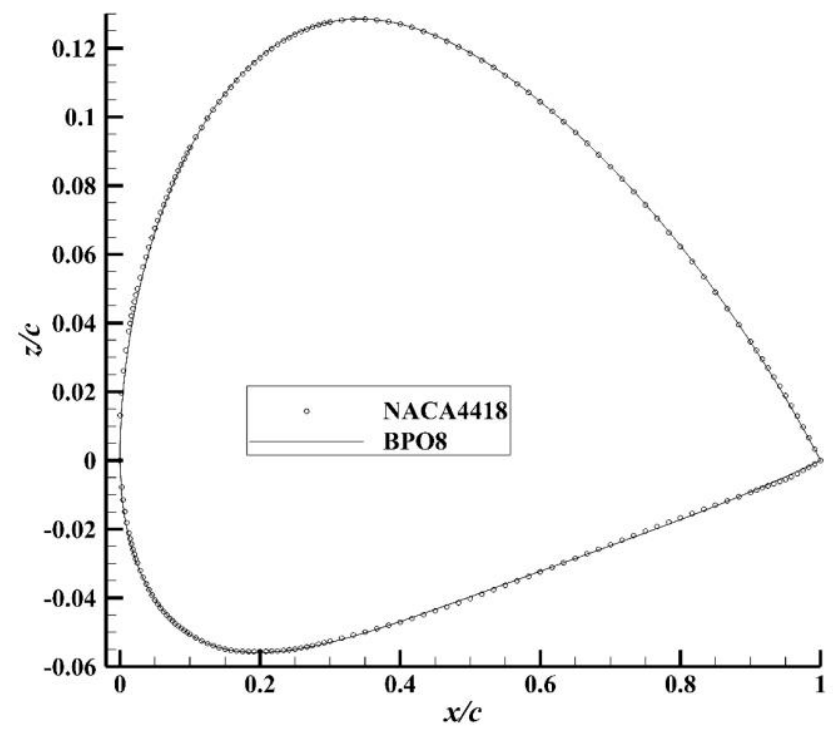

Figure 3: Comparison of the shape of the BPO8 airfoil and the pristine airfoil.

\subsection{Mixed function of index method}

Using the method proposed by Standish K J (Standish and van Dam, 2003) to increase the thickness without changing the

5 maximum thickness and the chord of the airfoil, the power function distribution is used to increase the thickness of the trailing edge of the airfoil to ensure the geometric continuity of the thickened airfoil (Jin et al., 2015).

Assuming the coordinates of the original airfoil are $\left(x_{0}, y_{0}\right)$, the coordinates of the thickened airfoil are $(x, y)$ :

$$
\left\{\begin{array}{ll}
x=x_{0}, y=y_{0} & \left(0 \leq x \leq x_{t}\right) \\
x=x_{0}, y_{u}=y_{0}+\delta_{1}\left(\frac{x-x_{t}}{c-x_{t}}\right)^{n} & \left(x_{t}<x \leq c\right) \\
x=x_{0}, y_{l}=y_{0}-\delta_{2}\left(\frac{x-x_{t}}{c-x_{t}}\right)^{n} & \left(x_{t}<x \leq c\right)
\end{array},\right.
$$

Where $x_{t}$ is the position of the maximum relative thickness of the airfoil, $\mathrm{m} ; \delta_{1}$ is the thickness of the trailing edge

10 of the upper airfoil, $\mathrm{m} ; \delta_{2}$ is the thickness of the trailing edge of the lower airfoil, $\mathrm{m} ; c$ is chord length, $\mathrm{m} ; n$ is the power exponent, taking into account the shape continuity and aerodynamics, $n=2$.

\subsection{Numerical methods and mesh division}

Figure 4 is a two-dimensional airfoil grid structure and a partial enlargement diagram. The airfoil is meshed using ICEM software. The left calculation domain is set to a semicircle with a radius of 10 times the chord length, and the calculation

15 domain on the right side is set to a rectangle with a length of 20 times the chord length. A C-shaped structured grid is used and local encryption processing is performed on the mesh around the airfoil. 

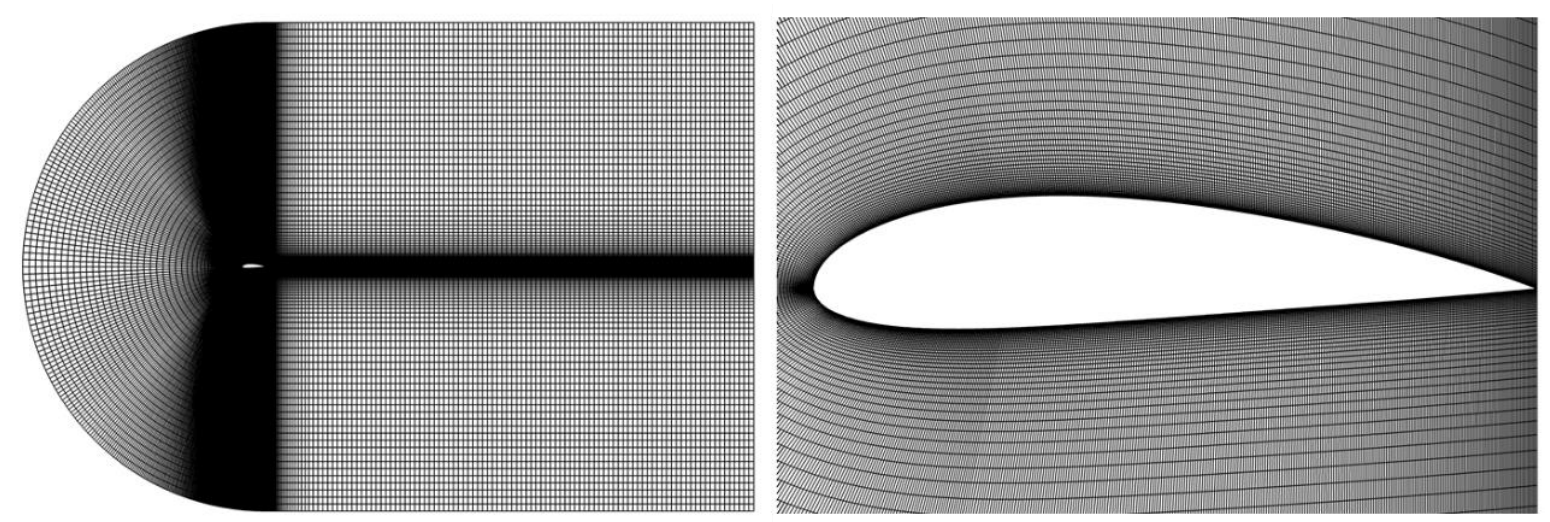

Figure 4: Overall grid diagram and grid partial enlargement diagram of NACA4418 airfoil.

In this paper, using the S-A model numerical calculation, discrete equations choose the finite volume method. The diffusion term uses a central difference format, and the convection term adopts second order upwind. The solution method adopts SIMPLE algorithm, and the boundary conditions are set as speed inlet and pressure outlet. The design wind speed is $29.21 \mathrm{~m} / \mathrm{s}$ and the Reynolds number is $2 \times 10^{6}$.

\section{Multi-Objective Genetic Algorithm Optimization}

\subsection{Optimization object}

For the wind turbine airfoil, the lift-to-drag ratio is the most important performance index, which has a great impact on

10 improving the operating efficiency of the wind turbine and the performance of the whole unit. In addition, ensuring the high lift starting characteristics and the gentle stalling characteristics of the airfoil are also important aspects in the operation of the wind turbine.

In this paper, the multi-objective genetic algorithm is used to select the maximum lift-to-drag ratio when operating at $6^{\circ}$ angle of attack, the maximum lift coefficient at $2^{\circ}$ low angle of attack, and the minimum drag coefficient at $17^{\circ}$ near stall

15 angle of attack. The airfoil multi-objective optimization problem can be expressed as:

Objective function:

$f=\min \left(-C_{l 2},-C_{l 6} / C_{d 6}, C_{d 17}\right)$,

Constraints: $C_{l 6} \geq C_{l}^{0}, C_{l}^{0}$ is the lift coefficient of the original airfoil at $6^{\circ}$ angle of attack, equals to 0.97 .

Optimization variable: For CST method, $b_{0, u}, b_{1, u}, b_{2, u}, b_{3, u}$ (upper surface weight factors), and $b_{0, l}, b_{1, l}, b_{2, l}, b_{3, l}$ (lower

20 surface weight factors) are chosen as the optimization variables. Their upper and lower limits are up and down $20 \%$ of the original airfoil weight factors. And Table 1 lists the disturbance range of the optimization variables. 


\begin{tabular}{ccc}
\hline variable & lower boundary & upper boundary \\
\hline$b_{0, u}$ & 0.243 & 0.365 \\
$b_{1, u}$ & 0.294 & 0.441 \\
$b_{2, u}$ & 0.238 & 0.357 \\
$b_{3, u}$ & 0.308 & 0.462 \\
$b_{0, l}$ & -0.244 & -0.163 \\
$b_{1, l}$ & -0.134 & -0.089 \\
$b_{2, l}$ & -0.104 & -0.070 \\
$b_{3, l}$ & -0.119 & -0.079 \\
\hline
\end{tabular}

Table 1: Disturbance range of optimization variables for CST method.

\subsection{Results and discussion}

Airfoils that satisfy the pareto optimal solution are further numerically calculated at an angle of attack of $0-17^{\circ}$. The main parameters of the optimized airfoils are listed in Table 2. The final optimized airfoil shape comparison is shown in Figure 5,

5 On the upper surface, the optimized airfoil differs very little from the original airfoil structure. The main change is that the leading edge radius is reduced, the airfoil is thinned, the upper surface is convex, and the lower surface is concave.

\begin{tabular}{ccccccccc}
\hline variable & $b_{0, u}$ & $b_{1, u}$ & $b_{2, u}$ & $b_{3, u}$ & $b_{0, l}$ & $b_{1, l}$ & $b_{2, l}$ & $b_{3, l}$ \\
\hline Opt1 & 0.35516 & 0.32819 & 0.29766 & 0.38823 & -0.16455 & -0.09329 & -0.07023 & -0.07903 \\
Opt2 & 0.34922 & 0.32802 & 0.29481 & 0.39348 & -0.16450 & -0.09329 & -0.07017 & -0.08375 \\
Opt3 & 0.33782 & 0.32819 & 0.29362 & 0.41967 & -0.16450 & -0.09382 & -0.07277 & -0.07915 \\
\hline
\end{tabular}

Table 2: The main parameters of the optimized airfoil.

Figure 6 is a comparison of pressure coefficient. It can be seen that the pressure coefficient in the front section of the upper surface is decreased, the rear section is basically consistent with the original airfoil, and the pressure coefficient on the lower surface is increased. In general, the difference in pressure between the upper and lower surfaces of the airfoil increases, resulting in an increase in the airfoil lift coefficient.

Figure 7 is a comparison of lift-to-drag ratio and Figure 8 is a comparison of lift coefficient. It can be seen that both the design and non-design conditions, the lift-to-drag ratio and lift coefficient of the airfoil are improved. Especially at $2^{\circ}$ angle of attack, the lift coefficient and lift-to-drag ratio increased by $12.36 \%$ and $12.20 \%$, respectively. In addition, the drag

15 coefficient at the large angle of attack decreased by $6.21 \%$, and the lift-to-drag ratio coefficient under other operating conditions also increased to varying degrees. This shows that the optimized airfoil has sensitive high lift start characteristics, good operating efficiency, and gentle stall performance. 
https://doi.org/10.5194/wes-2019-47

Preprint. Discussion started: 8 August 2019

(c) Author(s) 2019. CC BY 4.0 License.

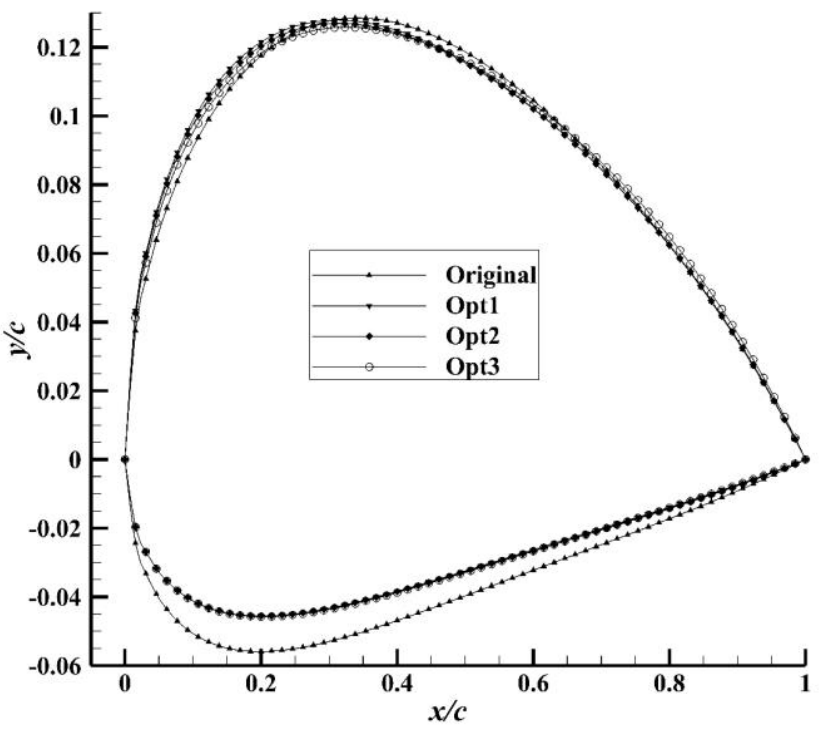

Figure 5: Comparison of the shape of optimized airfoils and the initial airfoil.

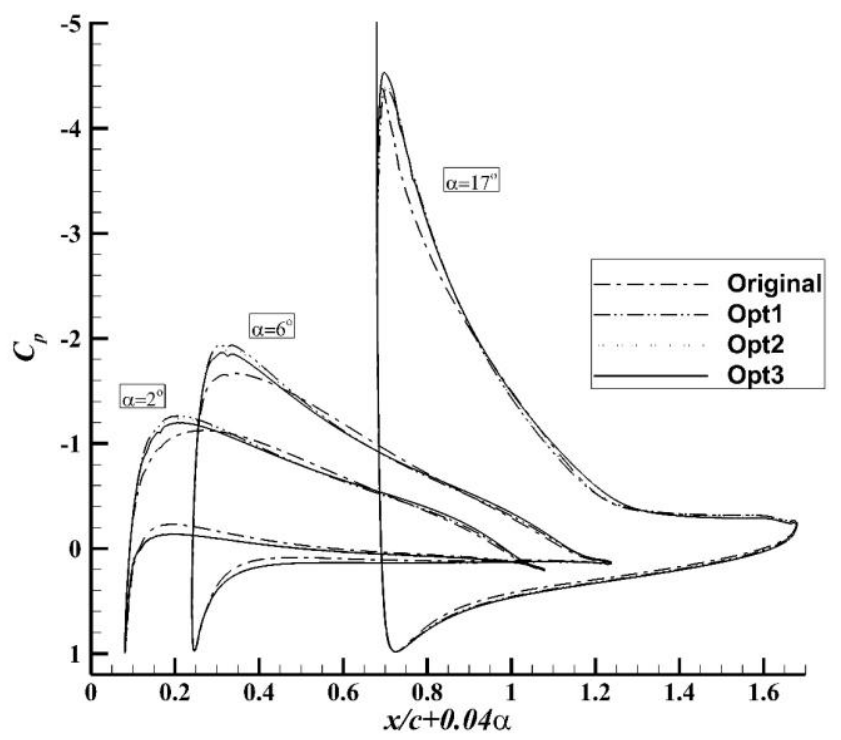

Figure 6: Comparison of pressure coefficients between optimized airfoils and the initial airfoil. 
https://doi.org/10.5194/wes-2019-47

Preprint. Discussion started: 8 August 2019

(c) Author(s) 2019. CC BY 4.0 License.

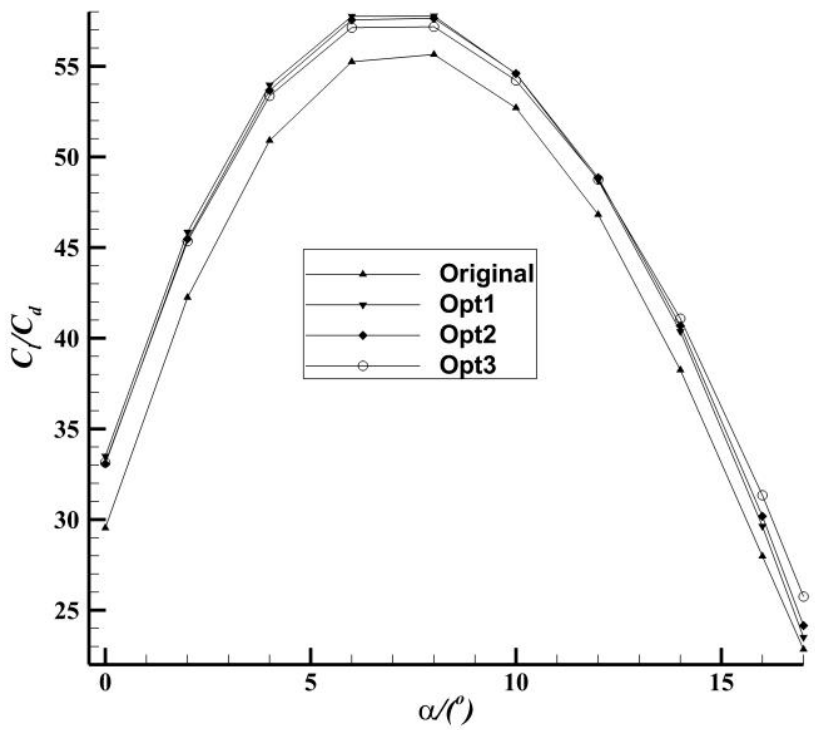

Figure 7: Comparison of lift-to-drag ratio coefficient between optimized airfoil and the initial airfoil.

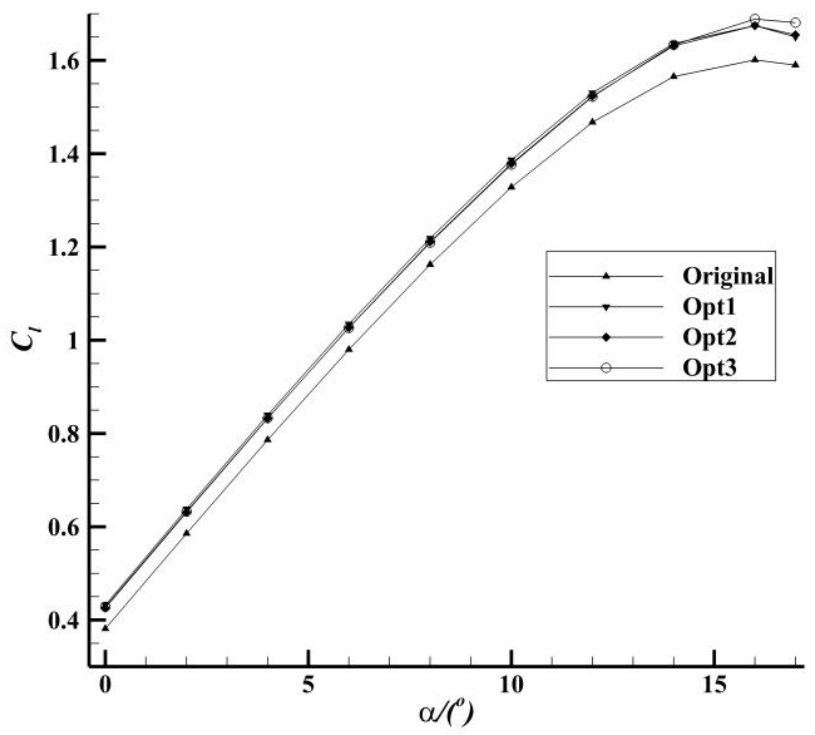

Figure 8: Comparison of lift coefficient between optimized airfoil and the initial airfoil. 


\section{Blunt Trailing Edge Optimization}

For the above analysis, the trailing edge of the Opt3 airfoil is thickened by the method shown in the formula (6). The optimization function is shown as equation (7). Taking the thickness of the trailing edge as the optimization variable with the constraint of not exceeding $0.05 c$. The orthogonal test method is used to establish the variable optimization space because of

5 the small number of variables. The lift coefficient and lift-to-drag ratio of the airfoil at $0-17^{\circ}$ angle of attack are investigated. In the analysis of 16 sets of different thickness airfoil calculation results, it can be found that the airfoil thickness increases, the lift coefficient increases, but the drag coefficient also increases greatly, so that the lift-to-drag ratio of the airfoil will be reduced to different extents.

The contrast between the blunt trailing airfoil and the Opt3 airfoil is shown in Figure 9. The front half of the airfoil has no change, the rear half of the airfoil has a gentle transition, the upper surface of the trailing edge is increased by $0.01 c$, and the lower surface is increased by $0.005 c$.

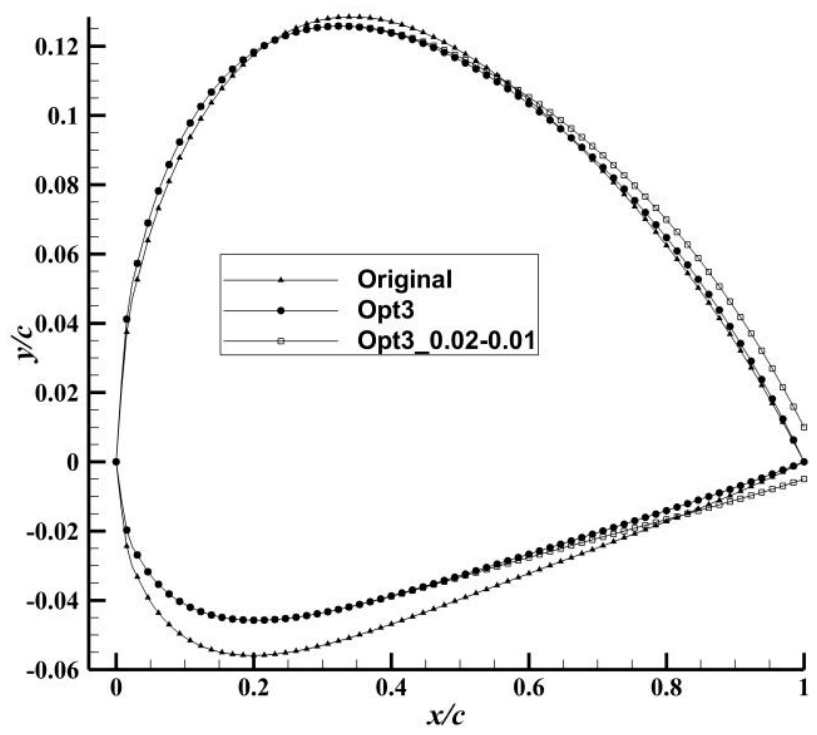

Figure 9: Comparison of the shape of optimized airfoils and the initial airfoil.

Figure 10 is a comparison of pressure coefficient. After the trailing edge is thickened, the pressure coefficient of the upper

15 and lower surfaces is basically the same as that of the Opt3 airfoil. The pressure coefficient of the upper surface of the airfoil is smaller near the trailing edge, and the pressure coefficient of the lower surface is larger. As the pressure difference increases, the lift coefficient further increases.

Figure 11 is a comparison of lift-to-drag ratio and Figure 12 is a comparison of lift coefficient. It can be seen that the blunt trailing-edge airfoil is better than the Opt3 airfoil under all operating conditions. Compared with the original airfoil, the lift coefficient and lift-to-drag ratio are increased by $23.55 \%$ and $19.01 \%$ at $2^{\circ}$ angle of attack, respectively, and the drag 
https://doi.org/10.5194/wes-2019-47

Preprint. Discussion started: 8 August 2019

(c) Author(s) 2019. CC BY 4.0 License.

(c) (i)

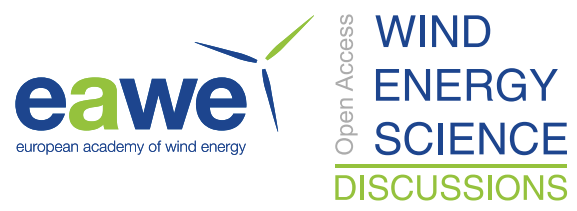

coefficient at $17^{\circ}$ angle of attack is reduced by $8.91 \%$. The blunt trailing edge airfoil has better lift-to-drag characteristics than the original airfoil and the Opt3 airfoil.

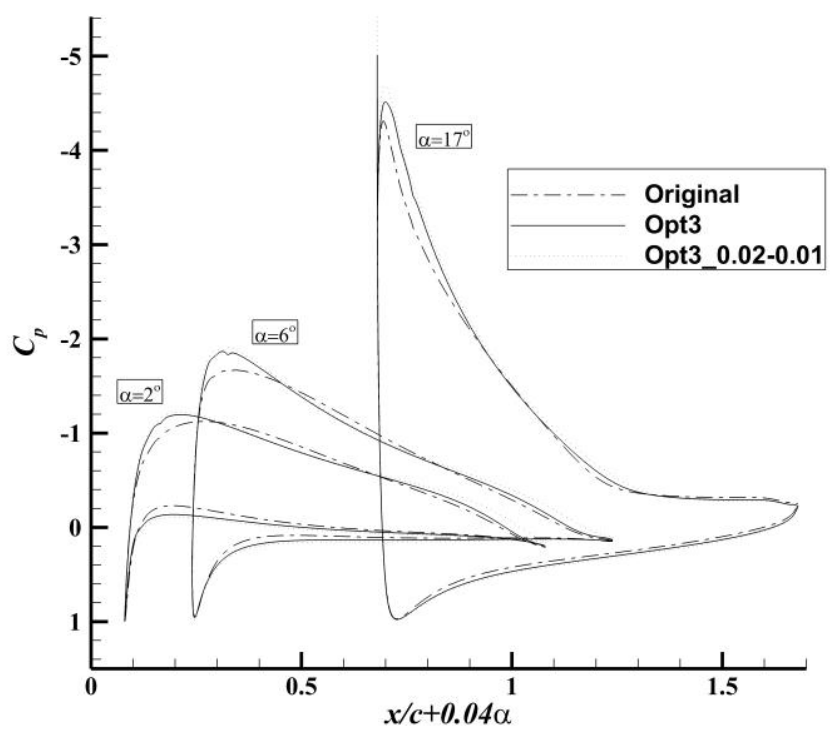

Figure 10: Comparison of pressure coefficients between optimized airfoil and initial airfoil.

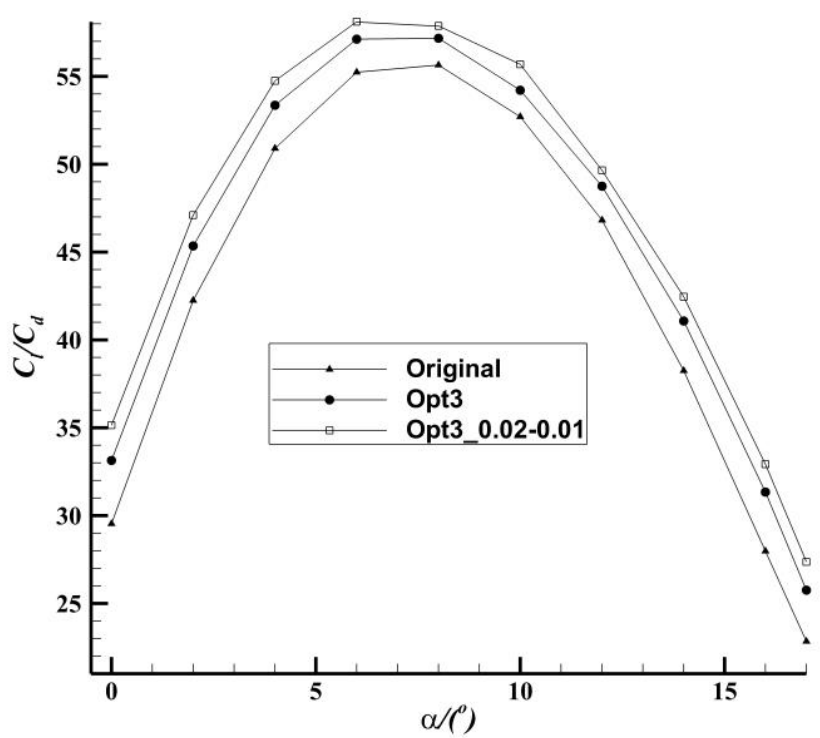

5

Figure 11: Comparison of lift-to-drag ratio coefficient between optimized airfoil and the initial airfoil. 
https://doi.org/10.5194/wes-2019-47

Preprint. Discussion started: 8 August 2019

(c) Author(s) 2019. CC BY 4.0 License.

(c) (i)
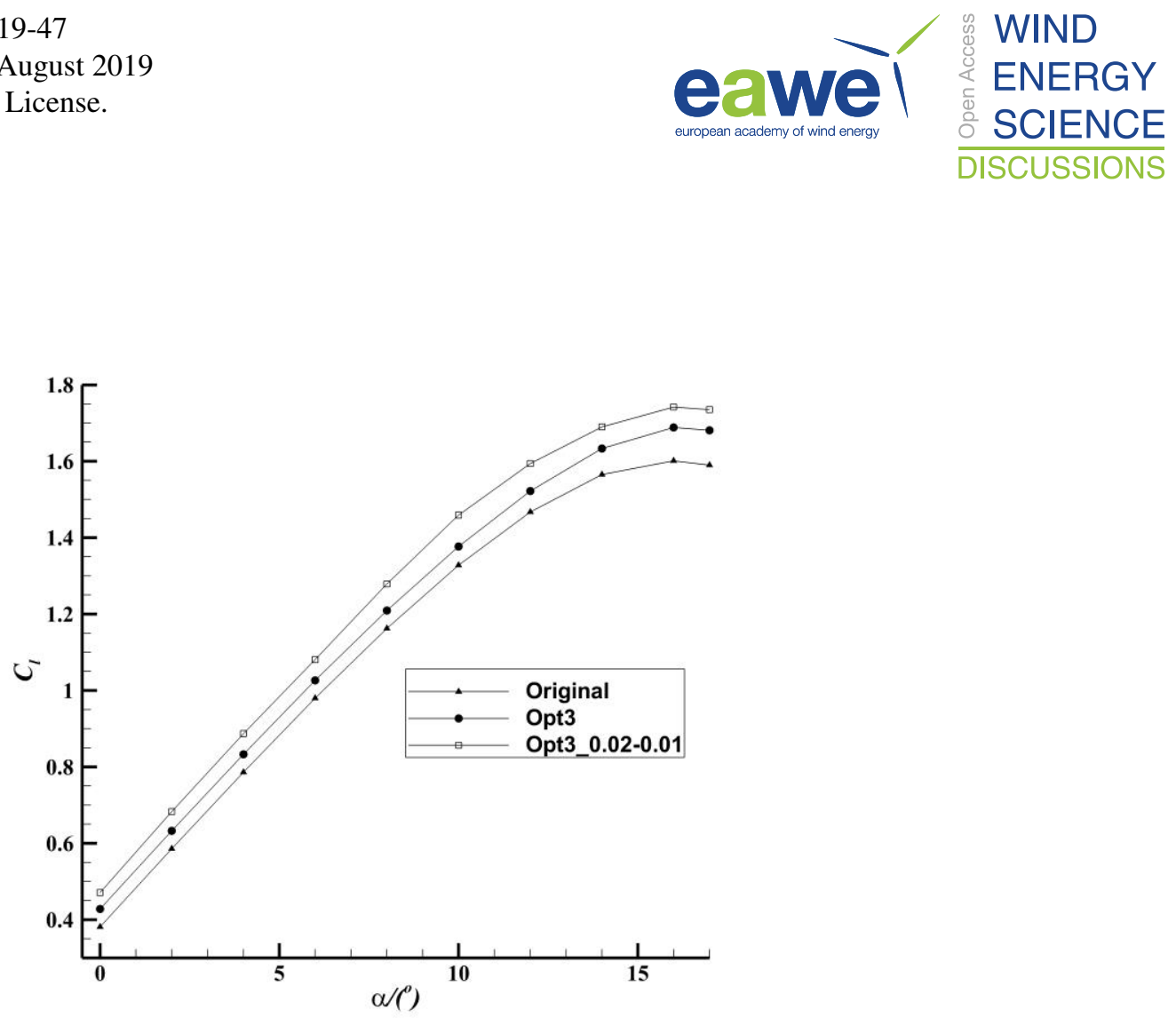

Figure 12: Comparison of lift coefficient between optimized airfoil and the initial airfoil.

Figure 13 is the comparison of three airfoil streamlines for different angles of attack. At an angle of attack of $14^{\circ}$, the vortex position of the blunt trailing-edge airfoil is behind that of the original airfoil and the Opt3 airfoil, and the airfoil's transition point is shifted back by $11.4 \%$ compared with the original airfoil. The vortex structure is also reduced. The $17^{\circ}$ angle of attack is the near stall angle of attack. Compared with the $14^{\circ}$ angle of attack, the vortex position and the transition point move forward. At this time, the flow condition is more complicated, the flow separation phenomenon is more obvious, and the risk of airfoil stall is increased.

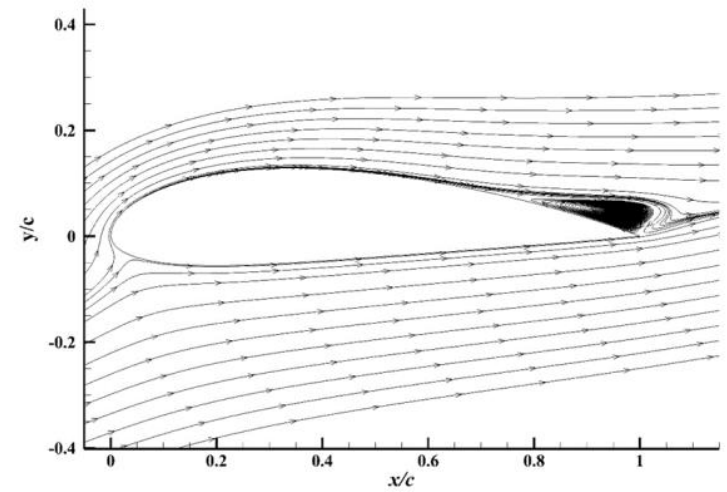

10 a. $14^{\circ}$ of Original airfoil

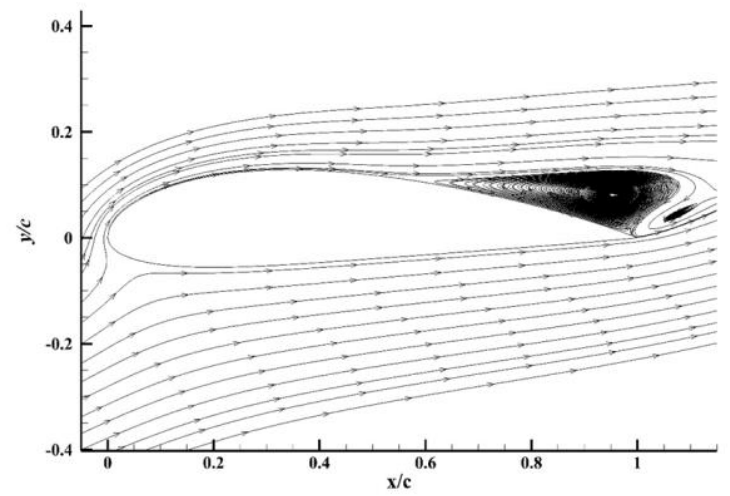

b. $17^{\circ}$ of Original airfoil 


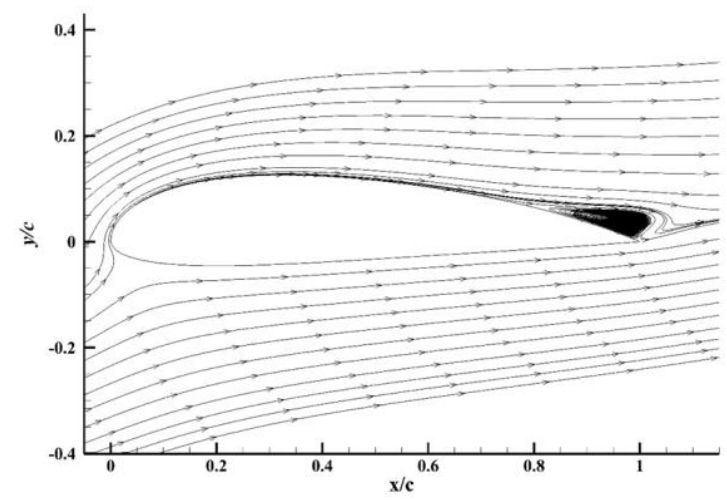

c. $14^{\circ}$ of Opt3 airfoil

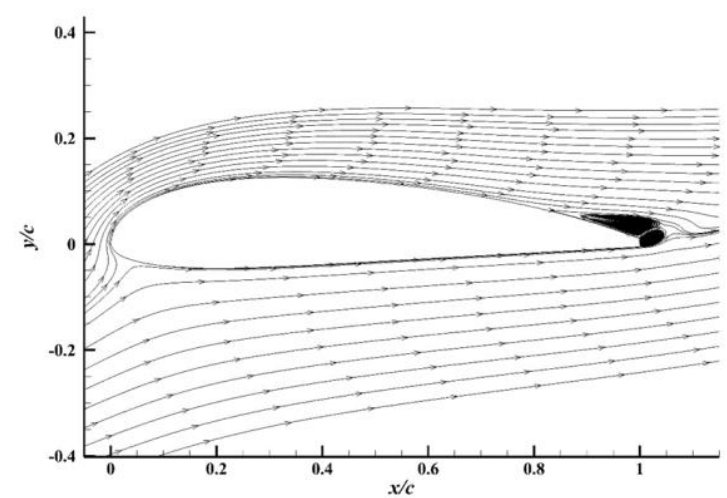

e. $14^{\circ}$ of Opt3_0.02-0.01 airfoil

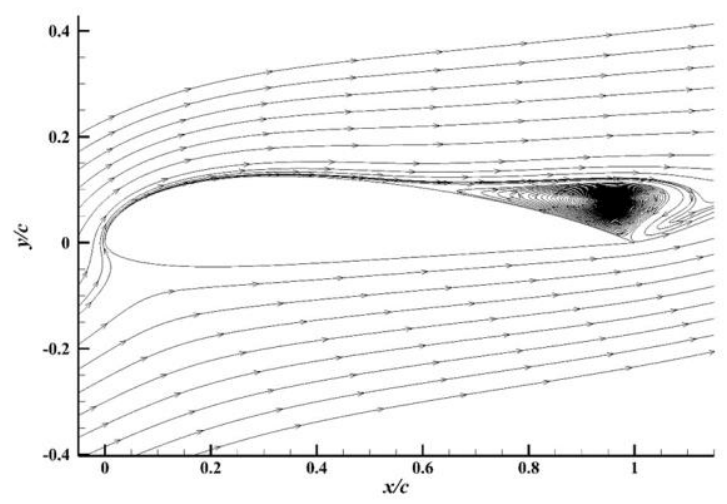

d. $17^{\circ}$ of Opt 3 airfoil

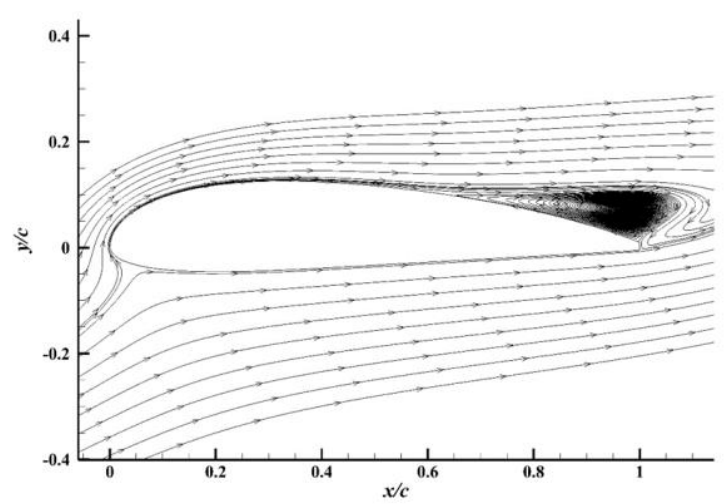

f. $17^{\circ}$ of Opt3_0.02-0.01 airfoil

5 Figure 13: Comparison of three airfoil streamlines for different angles of attack.

Compared with the original airfoil, after the airfoil is optimized and thickened, the transition point and the vortex position are shifted back by $13.7 \%$ and $3.4 \%$, respectively. The variation trend of the airfoil lift coefficient is explained from the flow level. The separation of the boundary layer of the pristine airfoil is delayed and the flow condition of the airfoil is improved.

\section{Conclusions}

10 The combination of the airfoil genetic algorithm and the trailing edge thickening technique achieves a blunt tailing edge airfoil with a large improvement in aerodynamic performance. Contributions of this research are summarized as follows:

1. Apply multi-objective genetic algorithm to the performance optimization of wind turbines, and call the corresponding software through script to realize wind turbine airfoil modelling, mesh division, numerical calculation, and performance 
https://doi.org/10.5194/wes-2019-47

Preprint. Discussion started: 8 August 2019

(c) Author(s) 2019. CC BY 4.0 License.

(c) (i)

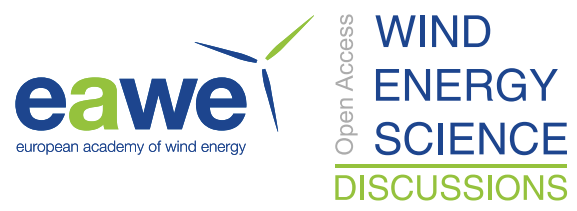

comparison automatically. It can save the airfoil optimization design time, and has great practical significance for the airfoil optimization design and the wind turbine blade design.

2. The lift coefficient of the blunt trailing edge airfoil is generally higher than that of the sharp tailing edge airfoil under the same working conditions, but the drag coefficient will also increase to a certain extent. Especially at high angles of attack,

5 the lift-to-drag ratio may be less than the original airfoil.

3. After the airfoil is optimized by algorithm and thickened by the blunt trailing edge, the transition point and the vortex position are shifted back, and the vortex structure is reduced to different extents, which improves the flow condition of the airfoil surface and delays the separation of the boundary layer.

\section{Data Availability}

10 NACA4418 data are available from UIUC Airfoil Coordinates Database; no other data are available.

\section{Author Contribution}

The creation of optimized process, data analysis and manuscript preparation were performed by Yong Peng. Jun Wang, Wei Wang and Guoqing Yin participated in technical discussions and manuscript revision.

\section{Competing Interests}

15 The authors declare that they have no conflict of interest.

\section{Acknowledgments}

The work was supported by the Double first-class research funding of China-EU Institute for Clean and Renewable Energy (ICARE-RP-2018-WIND-001). 
https://doi.org/10.5194/wes-2019-47

Preprint. Discussion started: 8 August 2019

(c) Author(s) 2019. CC BY 4.0 License.

\section{References}

Deng, L., Gao, Y. and Xiong, J.: Computational aerodynamic analysis of flatback airfoils by coupling N-S equations and transition prediction codes, Computational Methods and Experimental Measurements XV, 29(5), 45-54, doi:10.2495/cmem110051, 2011.

5 Farouki, R.: Legendre-Bernstein basis transformations, Journal of Computational and Applied Mathematics, 119(1-2), 145160, doi:10.1016/s0377-0427(00)00376-9, 2000.

Ferguson, J.: Multivariable Curve Interpolation, Journal of the ACM, 11(2), 221-228, doi:10.1145/321217.321225, 1964.

Fuglsang, P. and Bak, C.: Development of the Risø wind turbine airfoils, Wind Energy, 7(2), 145-162, doi:10.1002/we.117, 2004.

10 Hicks, R. and Henne, P.: Wing Design by Numerical Optimization, Journal of Aircraft, 15(7), 407-412, doi:10.2514/3.58379, 1978.

Jackson, K., Zuteck, M., van Dam, C., Standish, K. and Berry, D.: Innovative design approaches for large wind turbine blades, Wind Energy, 8(2), 141-171, doi:10.1002/we.128, 2005.

Jin, C., Xiaofeng, G., Yi, X. and Zhenye, S.: Optimization design method for the large thick wind turbine airfoils with a

15 blunt trailing edge, Journal of Harbin Engineering University, 36(7), 970-974, doi:10.3969/j.issn.1006-7043.201403022, 2015.

Kulfan, B. and Bussoletti, J.: "Fundamental" Parameteric Geometry Representations for Aircraft Component Shapes, 11th AIAA/ISSMO Multidisciplinary Analysis and Optimization Conference, doi:10.2514/6.2006-6948, 2006.

Law, S. and Gregorek, G.: Wind tunnel evaluation of a truncated NACA 64-621 airfoil for wind turbine applications, Final

20 Report Ohio State Univ Columbus, 1987.

Ma, R., Zhong, B. and Liu, P.: Optimization design study of low-Reynolds-number high-lift airfoils for the high-efficiency propeller of low-dynamic vehicles in stratosphere, Science China Technological Sciences, 53(10), 2792-2807, doi:10.1007/s11431-010-4087-0, 2010.

Sobieczky, H.: Parametric Airfoils and Wings, Notes on Numerical Fluid Mechanics (NNFM), 65, 71-87, doi:10.1007/978-

25 3-322-89952-1_4, 1999.

Standish, K. and van Dam, C.: Aerodynamic Analysis of Blunt Trailing Edge Airfoils, Journal of Solar Energy Engineering, 125(4), 479, doi:10.1115/1.1629103, 2003.

Tangler, J. and Somers, D.: NREL airfoil families for HAWTs, Nasa Sti/recon Technical Report N, 95, doi:10.2172/10106095, 1995.

30 van Rooij, R. and Timmer, W.: Roughness Sensitivity Considerations for Thick Rotor Blade Airfoils, Journal of Solar Energy Engineering, 125(4), 468, doi:10.1115/1.1624614, 2003.

van Rooij, R. and Timmer, W.: Roughness Sensitivity Considerations for Thick Rotor Blade Airfoils, Journal of Solar Energy Engineering, 125(4), 468, doi:10.1115/1.1624614, 2003. 
https://doi.org/10.5194/wes-2019-47

Preprint. Discussion started: 8 August 2019

(C) Author(s) 2019. CC BY 4.0 License.

(c) (1)

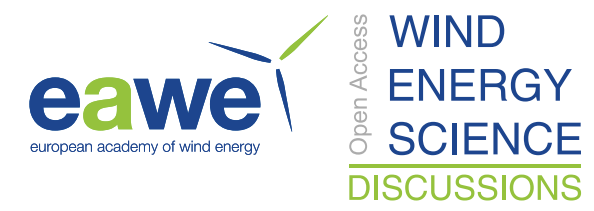

Yan, C.: Optimum Method and Inverse Method for Aerodynamic Design of Airfoil, Acta Aerodynamica Sinica, 17(1), 60-67, doi:10.3969/j.issn.0258-1825.1999.01.009, 1999.

Zhang, L.: Aerodynamic Influence of Different Trailing-edge Changing Methods to the Blunt Brailing-edge Airfoil, Journal of Engineering Thermophysics, 2009. 